\title{
Interventricular septal enlargement for left atrioventricular valve replacement with a larger prosthesis
}

\author{
Afksendiyos Kalangos, MD, PhD, Maurice Beghetti, MD, Dominique Vala, MD, Nicolas Murith, MD, and \\ Bernard Faidutti, MD, Geneva, Switzerland
}

From the Clinic for Cardiovascular Surgery, Division of Pediatric Cardiology, University Hospital of Geneva, Geneva, Switzerland.

Received for publication Aug 16, 2000; accepted for publication Oct 23, 2000.

Address for reprints: Afksendiyos Kalangos, MD, PhD, Clinic for Cardiovascular Surgery, University Hospital of Geneva, 24, rue Micheli-du-Crest, 1211 Geneva 14, Switzerland (E-mail: Afksendiyos.Kalangos@hcuge.ch).

J Thorac Cardiovasc Surg 2001;122:382-4

Copyright (C) 2001 by The American Association for Thoracic Surgery

$0022-5223 / 2001 \$ 35.00+0 \quad \mathbf{1 2 / 5 4 / 1 1 3 3 2 6}$

doi: $10.1067 / \mathrm{mtc} .2001 .113326$

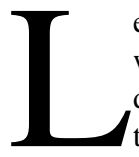
eft atrioventricular (AV) valve replacement in children with a small anulus in whom valve repair is not feasible may present a formidable challenge to the cardiac surgeon. In such cases, supra-annular implantation of the prosthesis ${ }^{1}$ or posterior enlargement of the anulus using several incision sites is of limited value. The purpose of this communication is to describe a new surgical procedure for enlarging the hypoplastic AV valve anulus, as illustrated by a case in which this technique was used for prosthetic valve replacement.

\section{Clinical Summary}

A 4-year-old boy was referred to our institution with the diagnosis of discordant AV and ventriculoarterial connection (corrected transposition) and associated large ventricular septal defect (VSD). He underwent pulmonary banding at the age of 7 months and closure of the VSD and pulmonary debanding in a different institution at the age of 2 years. The postoperative course after the second intervention was characterized by complete AV block requiring the implantation of a definitive pacemaker and the onset of left $\mathrm{AV}$ valve insufficiency, which became progressively severe within 2 years. At the time of referral to our institution, the patient was in New York Heart Association class IV with a 4/6 grade systolic murmur best heard on auscultation over the left fourth intercostal space. Chest radiography showed cardiomegaly with a cardiothoracic index estimated at $66 \%$. Transthoracic echocardiography confirmed the diagnosis of corrected L-transposition of the great arteries and revealed an Ebstein anomaly of the left AV valve associated with a narrow anulus and a supra-annular membrane. The degree of left AV valve regurgitation was grade IV and the mean transvalvular gradient was estimated at $20 \mathrm{~mm} \mathrm{Hg}$. Both ventricles showed signs of systolic and diastolic dysfunction. In the presence of mild right AV valve insufficiency, the left ventricular systolic pressure was estimated at $50 \mathrm{~mm} \mathrm{Hg}$. There was no residual leak through the VSD patch. The child was referred for a valvular operation. Cardiopulmonary bypass was established by cannulating the ascending aorta and the right atrium. Antegrade cold crytalloid cardioplegia and moderate hypothermia to $28^{\circ} \mathrm{C}$ were used for myocardial protection. The left AV valve was approached through an interatrial septotomy after excision of the supra-annular circular membrane, $3 \mathrm{~mm}$ in diameter. The left AV valve was tricuspid, with the septal and posterior leaflets located $1 \mathrm{~cm}$ below the anulus. Its repair was judged unfeasible because of small direct chordal attachments to the ventricular wall and septum, due to the absence of the anterior and septal papillary muscles. The leaflets were excised and the posterior anulus was incised at two sites to enlarge the small anulus. Despite these attempts to enlarge the anulus, only a $16-\mathrm{mm}$ mechanical valve sizer could be inserted, which was unsuitable for the body surface area of the child. The presence of AV block prompted the surgeon (A.K.) to enlarge the anulus by incising the interventricular septum. First, the posterior leaflet of the right AV valve was completely detached from the anulus. The left interatrial septal portion was then incised perpendicular to the interventricular septum, $2 \mathrm{~cm}$ away 

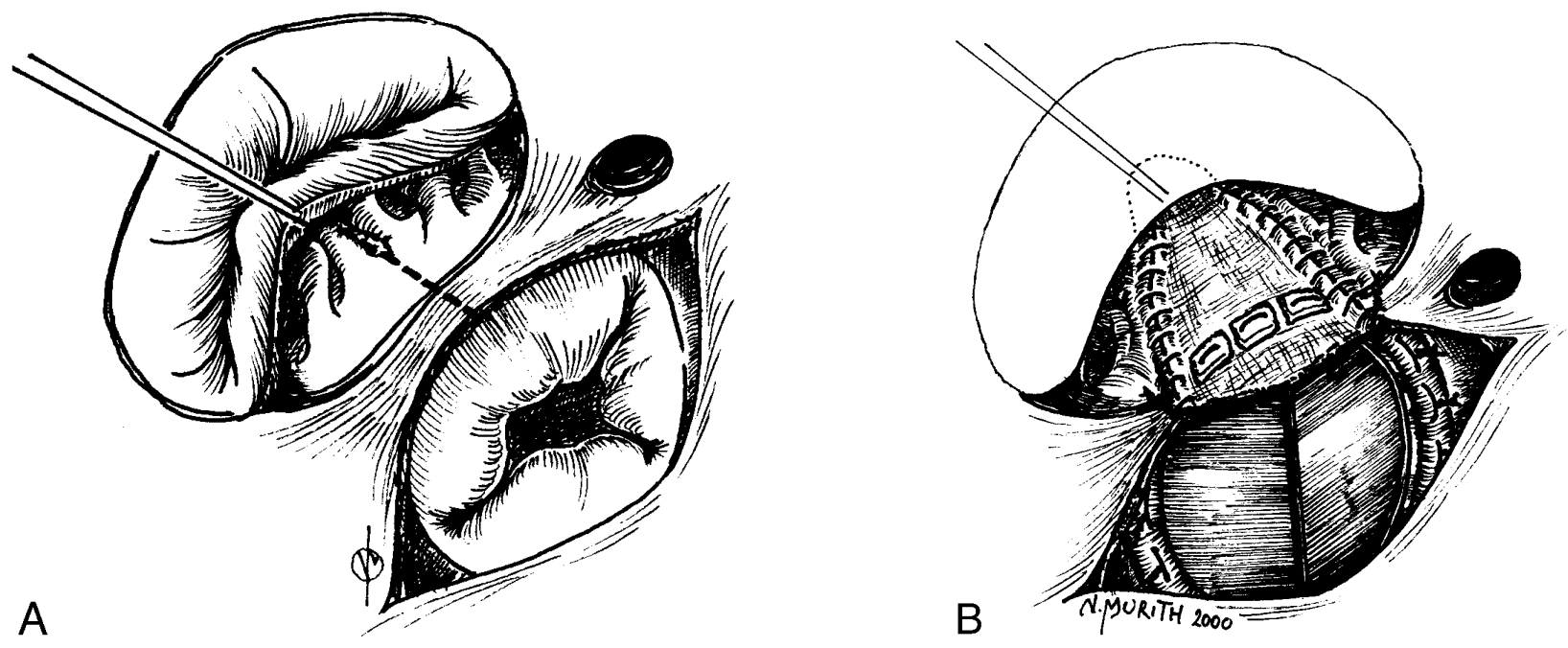

Figure 1. A and B, Sequence of the technique.

from the ostium of the coronary sinus. The incision was then extended $3 \mathrm{~cm}$ down into the middle portion of the interventricular septum (Figure 1, A). A 23-mm Carbomedics valve sizer (Sulzer Carbomedics, Inc [Division of Sulzer Medica], Austin, Tex) was placed at the level of the left AV valve anulus to determine the width of the anterior annular enlargement. A $3.5 \times 5 \mathrm{~cm}$ semi-ellipsoid bovine pericardial patch was cut and sutured to the borders of the interventricular septal defect, starting distally and going up to the anulus on both sides. A 23-mm Carbomedics prosthesis was then implanted in the anular position (Figure 1, B). The posterior leaflet of the right $\mathrm{AV}$ valve was then reattached to the patch, after the patch had been transversly plicated, $3 \mathrm{~mm}$ above the prosthesis, to a length equal to that of the detached portion of the posterior leaflet. Finally, the transected left part of the interatrial septum was reattached to the crest of the triangularly cut patch, and both portions of the interatrial septum were sewn together. Although this procedure involved an incision in the ventricular septum, the patient had nodal rhythm of 80 beats/min immediately after the operation. The patient had pulmonary hypertensive crises during the first 3 postoperative days, was extubated on the fourth postoperative day, and was discharged from the hospital 13 days later. He is currently doing well 8 months after the operation with a transprosthetic mean gradient of $4 \mathrm{~mm} \mathrm{Hg}$ and no signs of dysfunction of the prosthesis on echocardiography (Figure 2).

\section{Comment}

Although mitral valvulopathies associated with a narrow anulus that are not amenable to valve repair are uncommon, they are usually a source of hemodynamic problems if the size of the valvular prosthesis is not appropriate for the body surface area, particularly in growing children. Surgical literature in this field is very limited, probably because of the unresolved technical difficulties of adequately enlarging the left $\mathrm{AV}$ valve anulus. In fact, the left $\mathrm{AV}$ valve anulus is surrounded by anatomic structures that restrict

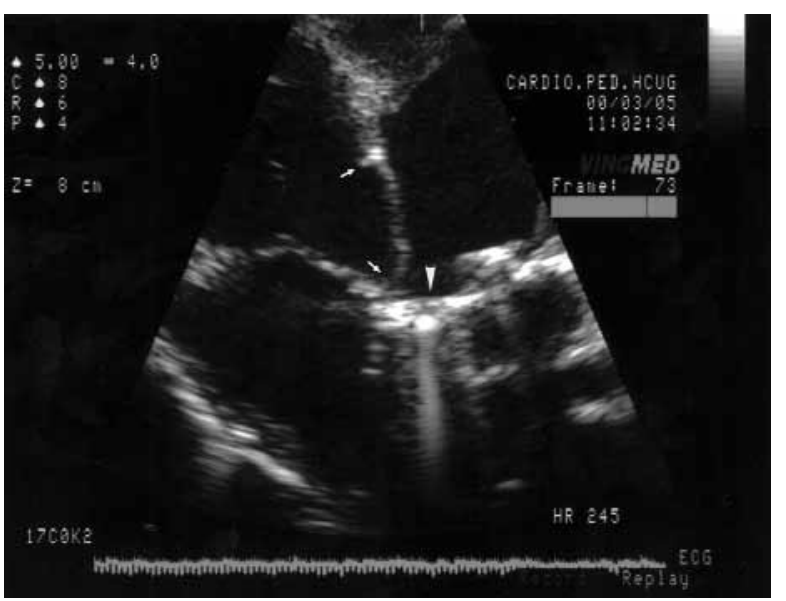

Figure 2. Transthoracic echocardiogram showing the interventricular septal enlargement using a pericardial patch (arrows) and the mechanical prosthesis (arrowhead) sutured onto the enlarged left $\mathrm{AV}$ anulus.

more aggressive enlargement procedures. An aggressive posterior annular enlargement can weaken the left AV junction and lead to type 1 ventricular rupture or expose the patient to complications related to the proximity of the coronary sinus and circumflex artery. Supra-annular or intra-atrial implantation of the prosthesis has the disadvantage to leave a stenotic area at the annular level. Contrary to our case, in which complete AV block was preexistent, the variability of the exact location of the AV node and the interventricular conduction path precludes the systematic use of interventricular septal enlargement. However, we believe that the development of preoperative or perioperative investigations aimed at precisely locating the conduction pathways could open new horizons and provide considerable security for the enlarge- 
ment of the interventricular septum for left AV valve replacement with an appropriately sized prosthesis. This AV valve version of the Konno procedure ${ }^{2}$ could be resorted to when no other safe or more traditional AV valve enlargement and implantation technique is possible.

\section{References}

1. Nataf P, Pavie A, Jault F, Bors V, Cabrol C, Gandjbakhch I. Intraatrial insertion of a mitral prosthesis in a destroyed or calcified mitral annulus. Ann Thorac Surg. 1994;58:163-7.

2. Konno S, Imai Y, Iida Y, Nakajima M, Tatsuno K. A new method for prosthetic valve replacement in congenital aortic stenosis associated with hypoplasia of the aortic valve ring. J Thorac Cardiovasc Surg. 1975;70:909-17. 